\begin{tabular}{|c|l|}
\hline Title & Activation of connective tissue growth factor gene by the c-Maf and Lc-Maf transcription factors \\
\hline Author(s) & Omoteyama, Kazuki; Ikeda, Hiromi; Imaki, Junko; Sakai, Masaharu \\
\hline Citation & $\begin{array}{l}\text { Biochemical and Biophysical Research Communications, 339/4), 1089-1097 } \\
\text { https://doi.org/40.1016/.bbrc.2005.11.119 }\end{array}$ \\
\hline Issue Date & $2005-12$-05 \\
\hline Doc URL & http://hdl.handle.net/2115989 \\
\hline Type & article (author version) \\
\hline File Information & BBRC339-4.pdf \\
\hline
\end{tabular}

Instructions for use 


\section{Activation of Connective Tissue Growth Factor Gene by the c-Maf and Lc-Maf Transcription Factors}

Kazuki Omoteyama ${ }^{1}$, Hiromi Ikeda ${ }^{1}$, Junko Imaki $^{2}$, and Masaharu Sakai ${ }^{1,3,4}$

${ }^{1}$ Department of Biochemistry, Graduate School of Medicine, Hokkaido University,

N14, W7, Kita-ku Sapporo 060-8638, Japan.

${ }^{2}$ Department of Anatomy, National Defense Medical College

3-2 Namiki, Tokorozawa, Saitama 359-8513, Japan.

${ }^{3}$ Department of Health Sciences, Hokkaido University School of Medicine,

N12, W5, Kita-ku, Sapporo 060-0812, Japan

${ }^{4}$ Corresponding Author,

Masaharu Sakai, Department of Health Sciences, Hokkaido University School of Medicine, N12, W5, Kita-ku, Sapporo 060-0812, Japan.

Tel \& FAX; +81-11-706-3407

E.mail: m_sakai@med.hokudai.ac.jp

Abbreviation: CTGF; connective tissue growth factor, TGF- $\beta$; transforming growth factor $\beta$, PCR; polymerase chain reaction, RT-PCR; reverse transcription and PCR, ChIP; chromatin immunoprecipitation, EST; expressed sequence tag. MEF; mouse embryonic fibroblast 


\begin{abstract}
The Maf family of transcription factors is expressed during development of various organs and tissues, and is involved in a variety of developmental and cellular differentiation processes. We previously found that $c$-maf and mafB are strongly expressed in hypertrophic chondrocytes during cartilage development. Connective tissue growth factor (CTGF) is also expressed in hypertrophic chondrocytes. Adenovirus mediated introduction of $c$-maf gene into the mouse fibroblast cell line C3H10T1/2 strongly induced CTGF expression. CTGF can be induced by TGF- $\beta$ via the SMAD pathway; however, the c-Maf could not induce TGF- $\beta$, nor could TGF- $\beta$ induce the c-Maf, suggesting that activation of CTGF by Maf is TGF- $\beta$ independent. Reporter transfection analysis using C3H10T1/2 cells shows that c-Maf stimulates a CTGF reporter gene. Lc-Maf, a splice variant of c-Maf containing an extra 10 amino acids in the carboxyl terminus, was a stronger inducer of the CTGF reporter gene than c-Maf. Chromatin immunoprecipitation analysis showed that c-Maf binds to the promoter region of the CTGF gene, indicating that Maf directly activates the CTGF gene. Gel mobility shift assays indicated that c-Maf binds to the region near the transcription initiation site of CTGF gene. Taken together, these data indicate that the CTGF gene is a target of c-Maf and Lc-Maf in cartilage development.
\end{abstract}

Key words: c-Maf, Lc-Maf, Connective tissue growth factor, Hypertrophic chondrocyte, Differentiation, Transcription control 


\section{Introduction}

The Maf family of basic-leucine zipper (bZip) transcription factors was first identified through the viral maf oncogene, an avian retroviral transforming gene [1]. There are two classes of Maf proteins, large Maf proteins such as c-Maf [2], MafB [3, 4], NRL [5], and L-Maf/MafA [6-8], and small Maf proteins, which lack the activation domain present in the large Mafs, including MafK, MafF [9, 10], and MafG [10]. The large Maf proteins can form either homodimers, or heterodimers with other bZip family members, such as Jun and Fos [3, 11, 12]. Once dimerized, Mafs bind to the Maf recognition element (MARE, -TGCTGAC(G)TCAGCA-), which overlaps with the 12-O-tetradecanoylphorbol 13-acetate responsive element (TRE) and the cAMP-responsive element (CRE) [11, 13].

Although the $c$-maf and mafB genes display oncogenic potential when over-expressed or translocated to the $\operatorname{IgH}$ locus $[1,14]$, recent reports support the involvement of these proteins in a variety of normal developmental and differentiation processes. The Maf family members appear to have significant and distinct functions in lens development, L-Maf and c-Maf are expressed in lens and induce the expression of several crystallin genes, and disruption of the $c$-maf gene in mice causes a failure in lens fiber formation $[6,15-18]$. The up-regulation of c-Maf by Pax6 is consistent with the role of $c$-maf in ocular development and differentiation [19]. Murine c-Maf regulates tissue specific expression of IL-4 and is involved in the cell fate determination of Th2 cells [20, 21]. c-Maf and MafA, activate glucagon and insulin gene expression in pancreatic islet $\alpha$ - and $\beta$-cells, respectively [8, 22, 23]. Additionally, the expression of $c$-maf mRNA is down-regulated during adipogenesis and up-regulated during myogenesis [24].

A recent study showed that c-Maf is highly expressed in hypertrophic chondrocytes and c-Maf is required for normal chondrocyte differentiation during endochondral bone development. Absence of c-Maf causes abnormal terminal differentiation of hypertrophic chondrocytes during endochondral bone development and fetal bone length decreases 
concomitantly with the decrease in the number of mature hypertrophic chondrocytes [25] [26].

Connective tissue growth factor (CTGF) CTGF is a member of the $\mathrm{CCN}$ ( $\underline{\mathrm{CTGF}}$, Cyr61, Nov) protein family which consists of CTGF, CRY61, Nov, WISP-1, WISP-2 and WISP-3, and a $36 \mathrm{kDa}$ cysteine rich protein $[27,28]$. CTGF is expressed in a variety of tissues, including hypertrophic chondrocytes[28, 29]. CTGF directly regulates the proliferation and differentiation of chondrocytes and osteoblasts $[28,30]$, stimulates angiogenesis in vivo [28, 31], and promotes extracellular matrix production in connective tissue [32]. Furthermore, CTGF plays an important role in the pathogenesis of various fibrotic disorders. It is over-expressed in scleroderma, keloids, and other fibrotic skin disorders, as well as in stromal rich mammary tumors, and in advanced atherosclerotic lesions [28, 33, 34]. CTGF is induced by transforming growth factor $\beta$ (TGF- $\beta$ ) through a TGF- $\beta$ response element (T $\beta$ RE) in the CTGF gene promoter $[28,35]$. A recent study also showed that TGF- $\beta$ activates SMADs and bound to the SMAD binding element (SBE) in the CTGF gene [36]. However, these studies examined CTGF gene regulation in pathogenic or artificially induced conditions. The regulation of normal developmental expression of CTGF, such as in hypertrophic chondrocytes, might be different from the TGF- $\beta$ mediated pathway [37].

In this study, we found that CTGF co-localized with c-Maf in hypertrophic chondrocytes, and we analyzed the possible relationship between Maf and CTGF gene regulation. The over-expression of c-Maf in the fibroblast cell line C3H10T1/2 strongly induced CTGF mRNA. Reporter transfection analysis, electrophoretic gel mobility shift analysis, and chromatin immunoprecipitation analysis each indicated that c-Maf and the Lc-Maf splice variant activate the CTGF gene through direct interactions with the CTGF gene promoter in a TGF- $\beta$ independent manner. 


\section{Materials and Methods}

In situ hybridization. - In situ hybridization was performed as described previously [25]. Pregnant mice were perfused through the aorta with $4 \%$ freshly prepared paraformaldehyde to fix embryos. Sections of E17.5 mouse limbs were hybridized with anti-sense RNA probes for $c$-maf and CTGF $\left(1 \times 10^{6} \mathrm{dpm} . / \mathrm{mL}\right)$. Slides were digested with RNase A $(20 \mu \mathrm{g} / \mathrm{mL})$ and washed sequentially in 2x SSC, $1 x$ SSC, $0.5 x$ SSC, and then incubated for 30 min in $0.1 \mathrm{x}$ SSC at $60^{\circ} \mathrm{C}$. The sections were exposed to X-ray film (Kodak X-omat) for 3 days, then dipped in NTB2 nuclear emulsion, (1:1 with water, Kodak), and exposed for 2 weeks. Radioactive RNA probes of $c$-maf, mafB and CTGF were synthesized with $\left[\alpha{ }^{35}\right.$ S] UTP by using the T7 RNA polymerase. In control experiments, adjacent sections were treated with RNase before hybridization or sense-strand RNA probes were used for hybridization.

Cloning of the mouse CTGF cDNA and genomic CTGF. - A mouse CTGF cDNA was amplified by RT-PCR using 5'-CTCCGTCGCAGGTCCCATCA-3' and 5'-CAGGCTTGGCGATTTTAGGT-3' as primers and cloned into the pBluescript II vector (Stratagene, La Jolla, CA). A mouse genomic library constructed in the EMBL-3 $\lambda$ phage vector was screened using mouse CTGF cDNA. Various restriction fragments of the clone were subcloned into pBluescript II and nucleotide sequences were determined by an ABI Prism dye terminator cycle sequencing kit and ABI 373S DNA sequencer (Applied Biosystems, Foster city, CA).

Construction of plasmids. - Various DNA fragments from the CTGF genomic clone were prepared by restriction cleavage or PCR and inserted into the promoter-less luciferase plasmid vector pGVB2 (Nippon Gene, Toyama, Japan). To analyze the enhancer activity of various regions of the CTGF gene, the fragments were inserted into the pGVB2 vector, which contains the rat glutathione S-transferase P (GST-P) gene promoter, -50 to $+36 \mathrm{bp}$, a minimal promoter containing a TATA box and a GC box [38]. Expression vectors of c-Maf have been described previously [25]. cDNA for rat Lc-maf, a splicing variant of $c$-maf, was cloned as 
followed. A rat EST (GenBank accession number; BF399640) containing the 3'-end of c-maf mRNA open reading frame contiguous to the coding sequence of Lc-Maf [39] was identified by a data base search. From this nucleotide sequence information, we designed the Lc-maf primers, 5'-ACACGTCCTGGAGTCGGAGA-3' and 5'-CCCACGGAGCATTTAACAAG-3', and successfully amplified rat $L c$-maf cDNA encoding the C-terminal region of Lc-Maf by RT-PCR. The 3'-terminal region of Lc-maf cDNA was ligated to the 5'-region of $c$-maf cDNA. These expression vectors were constructed in pcDNA3.1 (Invitrogen, Carlsbad, CA).

Cell culture and transient transfection analyses. - The mouse fibroblast cell line C3H10T1/2 was obtained from the Japanese Collection of Research Bioresources (Tokyo, Japan), and maintained in Dulbecco's modified minimal essential medium (DMEM; Nissui, Tokyo, Japan) supplemented with $10 \%$ fetal bovine serum (FBS). TGF $\beta$ treatments of $\mathrm{C} 3 \mathrm{H} 10 \mathrm{~T} 1 / 2$ cells were performed according to Chen [40]. The cells were serum starved for $18 \mathrm{~h}$ before being treated with 0.1 or $10 \mathrm{ng} / \mathrm{mL}$ TGF- $\beta$ (Sigma, St. Louis, MO) and harvested after $24 \mathrm{~h}$. Transient transfections were performed as described previously [24]. Briefly, $24 \mathrm{~h}$ before transfection, the cells were plated at a density of $1 \times 10^{5}$ cells/35-mm plate. C3H10T1/2 cells were co-transfected with a total of $2 \mu \mathrm{g}$ DNA, which included $0.5 \mu \mathrm{g}$ reporter plasmid, $0.2 \mu \mathrm{g}$ $\beta$-galactosidase expression plasmid (pSV $\beta$-gal; Promega, Madison, WI) as an internal control, various amounts of expression plasmid and pUC18 DNA. After $45 \mathrm{~h}$ incubation, cells were harvested and assayed for luciferase activity using a luciferase assay kit (Nippon Gene, Toyama, Japan) and for $\beta$-galactosidase activity. Luciferase activity was normalized to $\beta$-galactosidase activity and all experiments were repeated at least three times.

Adenovirus mediated introduction of Maf genes - Replication deficient adenoviruses carrying c-maf (AxCALNLrmaf2) [25] and Cre genes (AxCANCre) were provided by the RIKEN DNA Bank (Tsukuba, Japan). These genes are driven by the CAG promoter [41]. The $c-m a f$ gene is bracketed by the loxP element and expression is controlled by the Cre-loxP system. C3H10T1/2 cells were seeded at $24 \mathrm{~h}$ before infection and were co-infected for $1 \mathrm{~h}$ 
with various multiplicities of infection (MOI) of adenoviruses harboring a maf gene and the Cre gene in $0.5 \mathrm{~mL}$ medium containing 5\% FBS. Following co-infection, cells were fed 1.5 $\mathrm{mL}$ medium containing 10\% FBS. After $48 \mathrm{~h}$, cells were harvested for RNA or protein extraction.

RNA analysis - Total cellular RNA from C3H10T1/2 cells and tissues was prepared using an RNA extraction kit (Nippon Gene). To prepare an anti-sense RNA probe, cDNA fragments were subcloned into pBluescript II and synthesized by either T7 or T3 RNA polymerase (Takara, Kyoto, Japan) with $\left[\alpha_{-}{ }^{32} \mathrm{P}\right]$ UTP in vitro. The RNase protection analysis was performed as described previously [24].

Western blot analysis- Proteins were separated by SDS-polyacrylamide gel electrophoresis and transferred to a polyvinylidene difluoride membrane filter (Millipore, Bedford, MA). The membrane was incubated with anti-c-Maf serum $(1: 10,000)$ or anti-CTGF antibody $(1: 3,000)$ (Abcam, Cambridge, UK). After washing, the membrane was incubated with horseradish peroxidase-conjugated anti-rabbit IgG (Amersham-Pharmacia Biotech, Arlington, IL, 1:10,000) and the immunoreactive bands were detected using an enhanced chemiluminescence system (Amersham-Pharmacia Biotech).

ChIP analysis. - ChIP analysis was carried out as described previously [42, 43]. Briefly, cells were fixed in $1 \%$ formaldehyde and washed twice with phosphate-buffered saline containing protease inhibitors ( $1 \mathrm{mM} \mathrm{PMSF}, 1 \mu \mathrm{g} / \mathrm{mL}$ aprotinin, $1 \mu \mathrm{g} / \mathrm{mL}$ pepstatin). DNA of the fixed cells were sheared by sonication and diluted 10 -fold with IP buffer $(0.01 \%$ SDS, 1.1\% Triton X-100, 1.2 mM EDTA, 16.7 mM Tris- $\mathrm{HCl}, \mathrm{pH} 8.1,167 \mathrm{mM} \mathrm{NaCl})$. The samples were pre-cleared using Protein A-Sepharose (Pharmacia Biochemicals, Stockholm, Sweden) containing $20 \mu \mathrm{g} / \mathrm{mL}$ sheared salmon sperm DNA and $1 \mathrm{mg} / \mathrm{mL}$ BSA) and then incubated with an anti-c-Maf antibody or rabbit pre-immune serum for $16 \mathrm{~h}$ at $4^{\circ} \mathrm{C}$. Immune complexes were precipitate with Protein A Sepharose and washed sequentially with wash buffer-1 (0.1\% SDS, $1 \%$ Triton X-100, 2 mM EDTA, 20mM Tris-HCl, pH8.1) containing 150mM NaCl, 
wash buffer- 1 containing $500 \mathrm{mM} \mathrm{NaCl}$, wash buffer-2 (0.25 M LiCl, $1 \%$ NP40, $1 \%$ sodium deoxycholate, $1 \mathrm{mM}$ EDTA, $10 \mathrm{mM}$ Tris- $\mathrm{HCl}, \mathrm{pH} 8.1)$ and TE (10 mM Tris- $\mathrm{HCl}, \mathrm{pH} 8.1,1$ mM EDTA). The complexes were eluted in $1 \%$ SDS, $0.1 \mathrm{M} \mathrm{NaHCO}_{3}$, and cross-linking was reversed by heating to $65^{\circ} \mathrm{C}$ for $4 \mathrm{~h}$. DNAs were purified and specific promoter regions were amplified by PCR using the following primers: CTGF (5'-GGATCCGGTGTGAGTTGATGAGG-3' and 5'-GAGTGGATCTGGCTGAGTCT-3'), and $\quad$-jun (5'-AGAAAGAAGGGCCCAACTGTAGG-3' 5'-ATCCAGCCTGAGCTCAACACTTA-3').

Preparation of nuclear extracts and EMSA - Electrophoretic gel mobility shift assays (EMSA) were performed using a nuclear extract from C3H10T1/2 cells over-expressing c-Maf. Nuclear extracts were prepared using a nuclear extract preparation kit (Sigma). Probes were synthesized by filling in the 5'-overhanging end of the appropriate restriction enzyme fragment of the CTGF gene using Klenow DNA polymerase I (Takara, Kyoto, Japan) and $\left[\alpha-{ }^{32} \mathrm{P}\right]-\mathrm{dCTP}$. EMSA analyses were performed essentially as described previously [24]. Briefly, approximately $2.5 \mu \mathrm{g}$ nuclear extract was incubated with $2.5 \mu \mathrm{g}$ poly $(\mathrm{dI} / \mathrm{dC})$ in binding buffer (20 mM Hepes, pH 7.9, 20 mM KCl, 1 mM EDTA, 5 mM DTT, 4 mM MgCl${ }_{2}$, $15 \%$ glycerol, and $100 \mu \mathrm{g} / \mathrm{mL}$ BSA) and ${ }^{32} \mathrm{P}$-labeled DNA probe. For supershift assays, anti-c-Maf rabbit polyclonal IgG (Santa Cruz Biotechnology, Santa Cruz, CA) was incubated with the nuclear extract for $1 \mathrm{~h}$ at $15^{\circ} \mathrm{C}$ prior to the binding reaction. Protein-DNA complexes were analyzed by $4 \%$ polyacrylamide gel electrophoresis in $25 \mathrm{mM}$ Tris-borate, $0.5 \mathrm{mM}$ EDTA buffer. 


\section{Results}

The expression of $c$-maf and CTGF mRNA in mouse embryonic limbs.

We have previously reported that $c$-maf is strongly expressed in hypertrophic chondrocytes in the cartilages of the adult femur epiphysis and in the ribs and limbs of E15 rat embryos. Cartilage tissues artificially induced by subcutaneous implantation of bone morphogenetic protein (BMP) in rat also expressed $c$-maf[25]. Takigawa and colleagues reported that CTGF is also expressed in hypertrophic chondrocytes [29, 30]. To confirm the co-expression of these genes in hypertrophic chondrocytes, we conducted an in situ-hybridization analysis in developing mouse limbs (Fig. 1). In the E17.5 embryos, c-maf and CTGF mRNAs were strongly detected and showed similar tissue localization. The mRNAs were restricted almost entirely to hypertrophic chondrocytes in cartilage.

\section{Adenovirus mediated introduction of $c$-maf gene induces CTGF expression in C3H10T1/2 cells.}

To investigate possible interactions between Mafs and CTGF expression, we have introduced the $c$-maf genes into $\mathrm{C} 3 \mathrm{H} 10 \mathrm{~T} 1 / 2$ cells by adenovirus mediated gene transfer. We used the C3H10T1/2 mesenchymal stem cell line because these cells are able to differentiate into chondrocytes following treatment with 5-aza-cytidine, an inhibitor of DNA methylation [44], and are commonly used to investigate the mechanisms of chondrocyte differentiation [45-47]. CTGF mRNA was measured in C3H10T1/2 cells that over-expressed c-maf. Co-infection of C3H10T1/2 cells with $c$-maf containing adenovirus and Cre-expression virus induced CTGF mRNA in an MOI dependent manner (Fig. 2A). Western blotting analysis confirmed the induction of CTGF protein by c-Maf (Fig. 2B). To examine the effect of c-Maf on CTGF expression in vivo, we analyzed CTGF mRNA expression in mouse embryonic fibroblast (MEF) cells, established from c-maf knockout mice (generously provided by Dr. L. H. Glimcher, Harvard Medical School). CTGF mRNA in E17.5 knockout MEF cells was 
significantly reduced compared to wild-type MEF cells from a liter mate (Fig. 2C). Infection of $c$-maf $f^{/-}$MEF cells with $c$-maf containing adenovirus restored CTGF mRNA level to that of the wild type. CTGF mRNA expression was not completely shut-off in the $c$-maf ${ }^{/-}$MEF cells, but it is likely that MafB, as well as other factors, may activate CTGF gene.

\section{The CTGF induction by $c$-maf is TGF- $\beta$ independent.}

Many previous reports indicate that CTGF expression was induced by TGF- $\beta$ along with SMAD and other pathways [36, 48, 49]. Therefore, we investigated whether CTGF induction by Mafs correlated with TGF- $\beta$ expression. TGF- $\beta$ mRNA levels did not change significantly in adenovirus infected cells expressing c-maf (Fig. 3A). Additionally, 10 ng/mL TGF- $\beta$ induced CTGF mRNA in C3H10T1/2 cells, but $c$-maf mRNAs were not detected (Fig. 3B). The control cells, kidney, expressed $c$-maf mRNAs. CTGF induction by TGF- $\beta$ was confirmed by western blotting analysis, but c-Maf was not detected (Fig. 3C). These results suggest that CTGF induction by c-Maf is independent of the TGF- $\beta$ pathway.

\section{Cloning of $L c$-maf cDNA, a long splice variant of $c$-maf.}

Alternative splicing generates two forms of $c$-maf mRNA, the original described short-form, c-maf, and a long form, Lc-maf. The long form, Lc-maf encodes an extra 10 amino acids at the carboxyl terminus of c-Maf; the termination codon (UGA) of the c-maf mRNA is converted to UGC (tyr) by alternative splicing and another 9 amino acids are encoded in this transcript (Fig. 4A). Huang et al. reported that Lc-Maf is expressed in chondrocytes and transactivation of the collagen gene by Lc-Maf is stronger than that of c-Maf in a reporter transfection assay [39]. Therefore, we have cloned the rat Lc-Maf cDNA and analyzed its ability to induce CTGF gene expression. Cloning of $L c$-maf by RT-PCR is described in the Materials and Methods. Lc-maf was successfully amplified from RNA from muscle, brain, the $3 \mathrm{Y} 1$ fibroblast cell line, and BMP induced cartilage tissues. From a data 
base analysis, we determined that the second exon encoding 10 carboxyl-terminal amino acids is located more than $12 \mathrm{~kb}$ downstream from the first exon (Fig. 4A). We have analyzed the expression of $c$-maf and $L c$-maf in various tissues by RNase protection analysis (Fig. 4B). The anti-sense RNA probe used was the $c$-maf/Lc-maf junction region of the anti-Lc-maf mRNA sequence $(-182 /+60$, relative to the $c$-maf termination codon, $\underline{\mathrm{U}} \mathrm{GA})$, and, therefore, $c$-maf and Lc-maf mRNAs protected short (182 b) and long (242 b) probe sequences, respectively. Lc-maf expression was relatively minor compared to c-maf in all tissues examined. The cartilage tissues induced by subcutaneous implantation of BMP [25] also weakly expressed Lc-maf mRNA. The muscle cells expressed a relatively higher proportion of $L c$-maf to $c$-maf mRNA than found in other tissues.

\section{Lc-Maf and c-Maf activate the CTGF promoter}

To clarify the molecular mechanism of CTGF gene activation by c-Maf, we performed a reporter transfection analysis using $\mathrm{C} 3 \mathrm{H} 10 \mathrm{~T} 1 / 2$ cells. A reporter construct having $0.8 \mathrm{~kb}$ of the 5'-upstream region of the CTGF gene fused to a luciferase gene was strongly activated by co-transfection with the c-Maf or Lc-Maf expression vectors (Fig. 5A). We analyzed up to 5 $\mathrm{kb}$ from the cap site of the CTGF gene and activation of the reporter by either Lc-Maf or c-Maf was not significantly different from the $0.8 \mathrm{~kb} 5$ ' flanking sequence (data not shown). Lc-Maf activation of the CTGF reporter was 2-3-fold stronger than activation by c-Maf. To identify the c-Maf response elements in the CTGF gene, we analyzed a series of 5'-deletion constructs. Figure 5A shows that all of the constructs having up to $-134 \mathrm{bp}$ from the cap site were activated by co-transfection with c-Maf or Lc-Maf, suggesting that this proximal region was responsible for activation.

Next, various regions of the 5'-flanking sequence, -820/-596, -599/-319, -322/-136 and $-139 /+37$ were fused to a heterologous promoter, the rat glutathione-S transferase minimal promoter region, containing TATA- and GC- boxes, and the luciferase gene [38]. 
Although all of the fragments were activated by c-Maf and Lc-Maf, the proximal promoter fragment, $-139 /+37$, was most highly activated. Next, we examined regions farther down-stream of the cap site. Figure 5B shows the results of activation in reporter constructs using $-82 /+212$ bp or $-82 /+29$ bp. The construct with $-82 /+126$ bp showed the strongest activation. However, when we deleted $+70-+126 \mathrm{bp}$, the activity was reduced by half, suggesting that this region contains some activation elements. These results suggest that multiple elements exist near the promoter region, $-82-+212$. This region did not contain a typical MARE sequence (-TGCTGACTCAGCA- or $\mathrm{TGCN}_{7-8} \mathrm{GCA}$ ), but similar sequences were identified; TGAGTCTTCTT at $+21, \mathrm{TCCN}_{8} \mathrm{GCA}$ at $+70, \mathrm{TGGN}_{8} \mathrm{GAC}$ at +123 and $\mathrm{TGCN}_{10} \mathrm{GCA}$ at +191 . However, none of these sequences alone strongly responded to $c$-maf co-transfection. These data suggest that each of these elements alone are not strong enough to bind c-Maf or Lc-Maf. The combination of multiple binding elements may contribute to the strong binding and activation by these transcription factors.

\section{c-Maf binds to the CTGF gene promoter region.}

To examine whether the induction of CTGF expression by c-Maf is a direct interaction to with CTGF gene in vivo, we performed a chromatin immunoprecipitation (ChIP) analysis using an anti-c-Maf antibody. Chromatin from C3H10T1/2 cells with or without $c$-maf-adenovirus infection was used for this experiment. The anti-c-Maf antibody precipitated the CTGF promoter region from the chromatin of $c$-maf-adenovirus infected C3H10T1/2 cells, but not from un-infected cells (Fig. 6A). As a negative control, we amplified $c$-jun, and no amplified DNA was detected in any of the ChIP samples. This result clearly indicates that c-Maf directly and specifically binds to the CTGF gene promoter in cells over-expressing c-Maf.

To further narrow-down the region interacts with c-Maf, we carried out EMSA using nuclear extracts from c-Maf over-expressing C3H10T1/2 cells. Five fragments of he promoter 
region were used as probes. Although multiple protein-DNA complexes were seen with most of the probes, inclusion of the anti-c-Maf antibody in the assay decreased the amount of the c-Maf specific band and resulted in the appearance of a super-shifted band with some probes (Fig. 6B). Inclusion of anti-c-Maf in assays with probe D resulted in a decrease in the specific c-Maf band and the appearance of a super shifted band. In the presence of probe $\mathrm{B}$, the super shifted band was very weak, but the band corresponding to the c-Maf specific complex decreased in the presence of the anti-c-Maf antibody. Probe A also bound c-Maf and weakly super shifted. In assay using probe $\mathrm{C}$, the c-Maf complex decreased slightly, but a super shifted band was not clearly detected. These results suggest that multiple elements in the CTGF promoter from -135 to +175 bp of transcription initiation sites bind to the c-Maf.

ChIP and EMSA analyses clearly indicate that c-Maf bind to the promoter region of the CTGF gene in vivo and in vitro. 


\section{Discussion}

The importance of CTGF in the development of cartilage tissues was demonstrated by the CTGF knockout mouse [31]. CTGF stimulated cell proliferation, matrix remodeling and angiogenesis during chondrogenesis. TGF- $\beta$ activates the CTGF gene through the TGF- $\beta$ responsive element and SMAD binding element located on the CTGF promoter. However, several lines of evidence suggest that CTGF is also activated by TGF- $\beta$ independent manner [37]. Here, we showed that the Maf transcription factor activates CTGF gene in a TGF- $\beta$ independent manner. Over expression of c-Maf gene strongly induced CTGF expression (Fig. 2) and a reporter transfection analysis indicated that the proximal promoter regions were responsible for that activation (Fig. 5). ChIP and EMSA analyses indicated that c-Maf and Lc-Maf bound near the promoter region of the CTGF gene in vivo and in vitro (Fig. 6).

Previous studies have identified several functional response elements in the promoter region of the CTGF gene. Grotendorst et al. identified a TGF- $\beta$ responsive element at -162 bp in the CTGF gene, but the factor binding to this element was not identified. In addition to this element, Holmes et al identified a SMAD binding sequence just up-stream of the TGF- $\beta$ response element. These TGF- $\beta$ responsive sites are located out-side of the Maf responsive sequence, as determined by reporter transfection analysis and EMSA (Fig. 5, 6). These data are consistent with the results showing that Maf activates the CTGF gene independently of TGF- $\beta$.

Multiple AP-1 binding sites were found in the 5'-flanking sequence of CTGF. Although the AP-1 binding sequence resembles the MARE sequence, these AP-1 sites were did not contribute significantly to activation by Maf in the reporter transfection analysis (Fig. 5). EMSA also showed that the fragments containing AP-1 sites did not bind to the c-Maf protein (data not shown). Reporter transfection analysis and EMSA using nuclear extracts from cells over-expressing c-Maf indicate the presence of multiple c-Maf responsive elements between -131 to +175 bp of the CTGF gene. However, a typical MARE sequence was not 
found in this region. Some related sequences were found, as described above, but we could not identify the exact binding site in this region by footprinting analysis using the nuclear extracts because of the formation of multiple complexes and weak binding in the EMSA experiment (Fig. 6B). Recombinant Maf protein also failed to bind these sequences in EMSA (data not shown). These results suggest the possibility that c-Maf binds to these regions as a heterodimer with an unknown partner. c-Maf is known to form heterodimer with bZip- or other transcription factors, and heterodimer formation alters the binding sequence specificity $[11,12,50]$. We previously reported that heterodimers of c-Maf and c-Fos bind to different sequence than the c-Maf homodimer [11]. Therefore, it is possible that c-Maf or Lc-Maf form heterodimers with some unknown factor and bind to the CTGF promoter region. In the anti-c-Maf super-shift analysis (Fig. 6B), the behaviors of shifted and super-shifted bands were somewhat different depending on the probes used. Shifted bands of probe $\mathrm{B}$ decreased in the presence of the antibody but super-shifted bands were not significantly increased, whereas super-shifted bands of probe D clearly increased in the presence of antibody. It is possible that there are different heterodimer partners of c-Maf and the DNA binding activity of the heterodimers is differentially affected by the antibody.

Recently, Friedman et al. reported that NRL, a member of the large Maf family, and possibly other large Maf factors including c-Maf strongly interact with TATA binding protein (TBP) through the conserved N-terminal transactivation domain [51]. c-Maf and TBP may form part of a pre-initiation complex that binds near the promoter region with relatively weak binding affinities.

CTGF mRNA expression in the MEF cells from c-maf knockout mice, examined by RNase protection analysis (Fig. 2C) tended to decrease, but the effect was not significant. In situ hybridization analysis of CTGF expression in cartilage of the embryonic femur found no obvious difference from that of wild type mice (data not shown). This effect may be due to complementation by other transcription factors. Since over expression of MafB also 
activated CTGF expression in fibroblasts (data not shown), it is possible that MafB complements the c-Maf deficiency. However, the c-maf and CTGF knockout mice share the common phenotypes, showing expanded hypertrophic chondrocytes in the E16-E17 embryo and reduced ossification in the center of the tibia [26, 31]. Taken together these results indicate that c-Maf has important roles in chondrocyte development thorough the control of CTGF gene expression.

Acknowledgements:

We thank Dr. L. H. Glimcher (Harvard Medical School) for providing c-maf knockout mice and Dr. I. Saito (Institute of Medical Science, Tokyo University) for providing Cre-expessing adenovirus. We also thank the Japanease Collection of Research Bioresouces and the RIKEN Gene Bank, for providing the C3H10T1/2 cell line and Maf-expressing adenoviruses, respectively. This work was supported by a grant from Saitama Medical School Research Center for Genomic Medicine and by grants-in-aid from the Minister of Education, Science, Sports and Culture, Japan. 


\section{References}

[1]. M. Nishizawa, K. Kataoka, N. Goto, K. T. Fujiwara, and S. Kawai, v-maf, a viral oncogene that encodes a "leucine zipper" motif, Proc Natl Acad Sci U S A 86 (1989) 7711-7715.

[2]. K. Kataoka, M. Nishizawa, and S. Kawai, Structure-function analysis of the maf oncogene product, a member of the b-Zip protein family, J Virol 67 (1993) 2133-2141.

[3]. K. Kataoka, K. T. Fujiwara, M. Noda, and M. Nishizawa, MafB, a new Maf family transcription activator that can associate with Maf and Fos but not with Jun, Mol Cell Biol 14 (1994) 7581-7591.

[4]. S. P. Cordes, and G. S. Barsh, The mouse segmentation gene kr encodes a novel basic domain-leucine zipper transcription factor, Cell 79 (1994) 1025-1034.

[5]. A. Swaroop, J. Z. Xu, H. Pawar, A. Jackson, C. Skolnick, and N. Agarwal, A conserved retina-specific gene encodes a basic motif/leucine zipper domain, Proc Natl Acad Sci U S A 89 (1992) 266-270.

[6]. H. Ogino, and K. Yasuda, Induction of lens differentiation by activation of a bZIP transcription factor, L-Maf, Science 280 (1998) 115-118.

[7]. M. Olbrot, J. Rud, L. G. Moss, and A. Sharma, Identification of beta-cell-specific insulin gene transcription factor RIPE3b1 as mammalian MafA, Proc Natl Acad Sci U S A 99 (2002) 6737-6742.

[8]. K. Kataoka, S. I. Han, S. Shioda, M. Hirai, M. Nishizawa, and H. Handa, MafA is a glucose-regulated and pancreatic beta-cell-specific transcriptional activator for the insulin gene, J Biol Chem 277 (2002) 49903-49910.

[9]. K. T. Fujiwara, K. Kataoka, and M. Nishizawa, Two new members of the maf oncogene family, mafK and mafF, encode nuclear b-Zip proteins lacking putative trans-activator domain, Oncogene 8 (1993) 2371-2380.

[10]. K. Kataoka, K. Igarashi, K. Itoh, K. T. Fujiwara, M. Noda, M. Yamamoto, and M. Nishizawa, Small Maf proteins heterodimerize with Fos and may act as competitive repressors of the NF-E2 transcription factor, Mol Cell Biol 15 (1995) 2180-2190.

[11]. Y. Matsushima-Hibiya, S. Nishi, and M. Sakai, Rat maf-related factors: the specificities of DNA binding and heterodimer formation, Biochem Biophys Res Commun 245 (1998) 412-418.

[12]. T. K. Kerppola, and T. Curran, Maf and Nrl can bind to AP-1 sites and form heterodimers with Fos and Jun, Oncogene 9 (1994) 675-684.

[13]. K. Kataoka, M. Noda, and M. Nishizawa, Maf nuclear oncoprotein recognizes sequences related to an AP-1 site and forms heterodimers with both Fos and Jun, Mol Cell Biol 14 (1994) 700-712.

[14]. M. Chesi, P. L. Bergsagel, O. O. Shonukan, M. L. Martelli, L. A. Brents, T. Chen, E. Schrock, T. Ried, and W. M. Kuehl, Frequent dysregulation of the c-maf 
proto-oncogene at $16 \mathrm{q} 23$ by translocation to an Ig locus in multiple myeloma, Blood 91 (1998) 4457-4463.

[15]. H. Ogino, and K. Yasuda, Sequential activation of transcription factors in lens induction, Dev Growth Differ 42 (2000) 437-448.

[16]. J. I. Kim, T. Li, I. C. Ho, M. J. Grusby, and L. H. Glimcher, Requirement for the c-Maf transcription factor in crystallin gene regulation and lens development, Proc Natl Acad Sci U S A 96 (1999) 3781-3785.

[17]. S. Kawauchi, S. Takahashi, O. Nakajima, H. Ogino, M. Morita, M. Nishizawa, K. Yasuda, and M. Yamamoto, Regulation of lens fiber cell differentiation by transcription factor c-Maf, J Biol Chem 274 (1999) 19254-19260.

[18]. B. Z. Ring, S. P. Cordes, P. A. Overbeek, and G. S. Barsh, Regulation of mouse lens fiber cell development and differentiation by the Maf gene, Development 127 (2000) 307-317.

[19]. M. Sakai, M. S. Serria, H. Ikeda, K. Yoshida, J. Imaki, and S. Nishi, Regulation of c-maf gene expression by Pax6 in cultured cells, Nucleic Acids Res 29 (2001) 1228-1237.

[20]. I. C. Ho, M. R. Hodge, J. W. Rooney, and L. H. Glimcher, The proto-oncogene c-maf is responsible for tissue-specific expression of interleukin-4, Cell 85 (1996) 973-983.

[21]. I. C. Ho, J. I. Kim, S. J. Szabo, and L. H. Glimcher, Tissue-specific regulation of cytokine gene expression, Cold Spring Harb Symp Quant Biol 64 (1999) 573-584.

[22]. N. Planque, L. Leconte, F. M. Coquelle, S. Benkhelifa, P. Martin, M. P.

Felder-Schmittbuhl, and S. Saule, Interaction of Maf transcription factors with Pax-6 results in synergistic activation of the glucagon promoter, J Biol Chem 276 (2001) 35751-35760.

[23]. K. Kataoka, S. Shioda, K. Ando, K. Sakagami, H. Handa, and K. Yasuda, Differentially expressed Maf family transcription factors, c-Maf and MafA, activate glucagon and insulin gene expression in pancreatic islet alpha- and beta-cells, J Mol Endocrinol 32 (2004) 9-20.

[24]. M. S. Serria, H. Ikeda, K. Omoteyama, J. Hirokawa, S. Nishi, and M. Sakai, Regulation and differential expression of the c-maf gene in differentiating cultured cells, Biochem Biophys Res Commun 310 (2003) 318-326.

[25]. M. Sakai, J. Imaki, K. Yoshida, A. Ogata, Y. Matsushima-Hibaya, Y. Kuboki, M. Nishizawa, and S. Nishi, Rat maf related genes: specific expression in chondrocytes, lens and spinal cord, Oncogene 14 (1997) 745-750.

[26]. H. E. MacLean, J. I. Kim, M. J. Glimcher, J. Wang, H. M. Kronenberg, and L. H. Glimcher, Absence of transcription factor c-maf causes abnormal terminal differentiation of hypertrophic chondrocytes during endochondral bone development, Dev Biol 262 (2003) 51-63.

[27]. B. Perbal, CCN proteins: multifunctional signalling regulators, Lancet 363 (2004) 62-64. 
[28]. M. Takigawa, T. Nakanishi, S. Kubota, and T. Nishida, Role of CTGF/HCS24/ecogenin in skeletal growth control, J Cell Physiol 194 (2003) 256-266.

[29]. T. Nakanishi, Y. Kimura, T. Tamura, H. Ichikawa, Y. Yamaai, T. Sugimoto, and M. Takigawa, Cloning of a mRNA preferentially expressed in chondrocytes by differential display-PCR from a human chondrocytic cell line that is identical with connective tissue growth factor (CTGF) mRNA, Biochem Biophys Res Commun 234 (1997) 206-210.

[30]. T. Fukunaga, T. Yamashiro, S. Oya, N. Takeshita, M. Takigawa, and T. Takano-Yamamoto, Connective tissue growth factor mRNA expression pattern in cartilages is associated with their type I collagen expression, Bone 33 (2003) 911-918.

[31]. S. Ivkovic, B. S. Yoon, S. N. Popoff, F. F. Safadi, D. E. Libuda, R. C. Stephenson, A. Daluiski, and K. M. Lyons, Connective tissue growth factor coordinates chondrogenesis and angiogenesis during skeletal development, Development 130 (2003) 2779-2791.

[32]. J. K. Crean, D. Finlay, M. Murphy, C. Moss, C. Godson, F. Martin, and H. R. Brady, The role of p42/44 MAPK and protein kinase B in connective tissue growth factor induced extracellular matrix protein production, cell migration, and actin cytoskeletal rearrangement in human mesangial cells, J Biol Chem 277 (2002) 44187-44194.

[33]. C. C. Chen, N. Chen, and L. F. Lau, The angiogenic factors Cyr61 and connective tissue growth factor induce adhesive signaling in primary human skin fibroblasts, $\mathbf{J}$ Biol Chem 276 (2001) 10443-10452.

[34]. T. Shakunaga, T. Ozaki, N. Ohara, K. Asaumi, T. Doi, K. Nishida, A. Kawai, T. Nakanishi, M. Takigawa, and H. Inoue, Expression of connective tissue growth factor in cartilaginous tumors, Cancer 89 (2000) 1466-1473.

[35]. G. R. Grotendorst, H. Okochi, and N. Hayashi, A novel transforming growth factor beta response element controls the expression of the connective tissue growth factor gene, Cell Growth Differ 7 (1996) 469-480.

[36]. A. Holmes, D. J. Abraham, S. Sa, X. Shiwen, C. M. Black, and A. Leask, CTGF and SMADs, maintenance of scleroderma phenotype is independent of SMAD signaling, $\mathrm{J}$ Biol Chem 276 (2001) 10594-10601.

[37]. I. E. Blom, R. Goldschmeding, and A. Leask, Gene regulation of connective tissue growth factor: new targets for antifibrotic therapy?, Matrix Biol 21 (2002) 473-482.

[38]. M. Sakai, A. Okuda, and M. Muramatsu, Multiple regulatory elements and phorbol 12-O-tetradecanoate 13-acetate responsiveness of the rat placental glutathione transferase gene, Proc Natl Acad Sci U S A 85 (1988) 9456-9460.

[39]. W. Huang, N. Lu, H. Eberspaecher, and B. De Crombrugghe, A new long form of c-Maf cooperates with Sox 9 to activate the type II collagen gene, J Biol Chem 277 (2002) 50668-50675.

[40]. M. M. Chen, A. Lam, J. A. Abraham, G. F. Schreiner, and A. H. Joly, CTGF expression is induced by TGF- beta in cardiac fibroblasts and cardiac myocytes: a 
potential role in heart fibrosis, J Mol Cell Cardiol 32 (2000) 1805-1819.

[41]. H. Niwa, K. Yamamura, and J. Miyazaki, Efficient selection for high-expression transfectants with a novel eukaryotic vector, Gene 108 (1991) 193-199.

[42]. V. Orlando, H. Strutt, and R. Paro, Analysis of chromatin structure by in vivo formaldehyde cross-linking, Methods 11 (1997) 205-214.

[43]. H. Ikeda, S. Nishi, and M. Sakai, Transcription factor Nrf2/MafK regulates rat placental glutathione S-transferase gene during hepatocarcinogenesis, Biochem J 380 (2004) 515-521.

[44]. S. M. Taylor, and P. A. Jones, Multiple new phenotypes induced in 10T1/2 and 3T3 cells treated with 5- azacytidine, Cell 17 (1979) 771-779.

[45]. H. Nochi, J. H. Sung, J. Lou, H. D. Adkisson, W. J. Maloney, and K. A. Hruska, Adenovirus mediated BMP-13 gene transfer induces chondrogenic differentiation of murine mesenchymal progenitor cells, J Bone Miner Res 19 (2004) 111-122.

[46]. V. Lefebvre, P. Li, and B. de Crombrugghe, A new long form of Sox5 (L-Sox5), Sox6 and Sox 9 are coexpressed in chondrogenesis and cooperatively activate the type II collagen gene, Embo J 17 (1998) 5718-5733.

[47]. A. Hoffmann, S. Czichos, C. Kaps, D. Bachner, H. Mayer, B. G. Kurkalli, Y. Zilberman, G. Turgeman, G. Pelled, G. Gross, and D. Gazit, The T-box transcription factor Brachyury mediates cartilage development in mesenchymal stem cell line C3H10T1/2, J Cell Sci 115 (2002) 769-781.

[48]. G. R. Grotendorst, Connective tissue growth factor: a mediator of TGF-beta action on fibroblasts, Cytokine Growth Factor Rev 8 (1997) 171-179.

[49]. A. Leask, A. Holmes, C. M. Black, and D. J. Abraham, Connective tissue growth factor gene regulation. Requirements for its induction by transforming growth factor-beta 2 in fibroblasts, J Biol Chem 278 (2003) 13008-13015.

[50]. S. P. Hedge, A. Kumar, C. Kurschner, and L. H. Shapiro, c-Maf interacts with c-Myb to regulate transcription of an early myeloid gene during differentiation, Mol Cell Biol 18 (1998) 2729-2737.

[51]. J. S. Friedman, H. Khanna, P. K. Swain, R. Denicola, H. Cheng, K. P. Mitton, C. H. Weber, D. Hicks, and A. Swaroop, The minimal transactivation domain of the basic motif-leucine zipper transcription factor NRL interacts with TATA-binding protein, $\mathbf{J}$ Biol Chem 279 (2004) 47233-47241. 


\section{Figure Legends}

Figure 1 Expression of CTGF and $c$-maf mRNA in the limbs of an E17.5 mouse embryo. The indicated mRNAs were detected by in situ hybridization in hypertrophic chondrocytes.

Figure 2 Induction of CTGF mRNA by adenovirus mediated over-expression of $c$-maf. (A) CTGF and $c$-maf mRNAs were detected by RNase protection analysis in $10 \mu \mathrm{g}$ of total RNA from $c$-maf (Ad/c-maf) expressing adenovirus infected C3H10T1/2 cells. The MOIs are indicated above the samples. The cultures were co-infected with the same dose of Cre expressing adenovirus. (B) CTGF and c-Maf proteins in c-Maf over-expressing cells (100 $\mu$ g cell lysate) were detected by Western blotting. (C) CTGF mRNA was analyzed from the $c$-maf knockout- and wild type-mice E17.5 MEF cells, as in (A).

Figure 3 CTGF gene activation by Maf is TGF- $\beta$ independent. The mRNAs of TGF- $\beta$ and CTGF were determined in c-Maf over-expressing cells by RNase protection analysis, as described in Fig. 2A. Ten micrograms of total RNA was used for each analysis. (B) $c$-maf and CTGF mRNAs were analyzed in TGF- $\beta$ treated C3H10T1/2 cells, as described in the Materials and Methods. Rat kidney RNA was used as a positive control. (C) CTGF and c-Maf proteins were detected by Western blotting analysis. Recombinant c-Maf/maltose binding protein fusion protein was used as a positive control (c-Maf/Mal)

Figure 4 Cloning and expression of Lc-Maf mRNA. (A) Schematic representation of rat $c$-maf gene, and the $c$-maf and Lc-maf mRNAs. The nucleotide sequences encoding the c-terminal region of $c$-maf and $L c$-maf are shown. The probe used for RNase protection analysis is indicated by the horizontal bar. The closed and open arrowheads indicate the transcription and translation initiation sites, respectively. (B) Expression of $c$-maf and Lc-maf mRNA in various tissues. Ten micrograms of total RNA from the indicated tissues were analyzed. Liv: liver, Br: brain, Mus: muscle, BMP: chondrocyte tissues induced by subcutaneously implanted BMP in rat.

Figure 5 Activation of the CTGF promoter by c-Maf and Lc-Maf. (A) The indicated CTGF promoter constructs, containing 5'-deletions or internal deletions, were co-transfected with or without c-Maf or Lc-Maf expression plasmids into C3H10T1/2 cells and luciferase activity was measured in cell extracts collected 45 $\mathrm{h}$ after transfection. The structure of the CTGF gene is indicated schematically in the upper panel. Transcription and translation initiation sites are indicate by closed and open horizontal arrows, respectively. (B) Transfection analysis of regions 
proximal to and down-stream of the cap site. The indicated constructs were analyzed as described in (A).

Figure 6 c-Maf binds to the CTGF gene promoter in cells over-expressing c-Maf in vivo and in vitro. (A) Chromatin immunoprecipitation analyses were performed with anti-c-Maf antibody in C3H10T1/2 cells with (C3H10T1/2-Ad/c-maf) or without (C3H10T1/2) Ad/c-Maf infection, as described in the Materials and Methods. The DNAs were extracted from total sonicated nuclei (Input), Protein A without antibody (-), Protein A with antibody (Anti-c-Maf), or with pre-immune serum (Pre-Immune). Specific promoter regions of the CTGF and c-jun genes were amplified by PCR (33 cycles), separated on 5\% polyacrylamide gels and stained with ethidium bromide. (B) EMSA analysis of c-Maf binding to CTGF promoter fragments. Nuclear extracts of C3H10T1/2 cells over-expressing c-Maf were incubated with the indicated CTGF promoter fragments, with (+) or without (-) anti-c-Maf antibody. Transcription and translation initiation sites are indicate by closed and open horizontal arrows, respectively. 


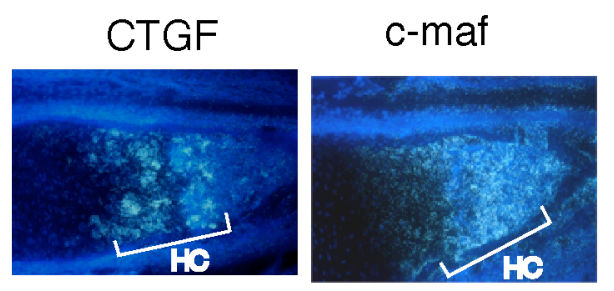

Fig. 1 
A

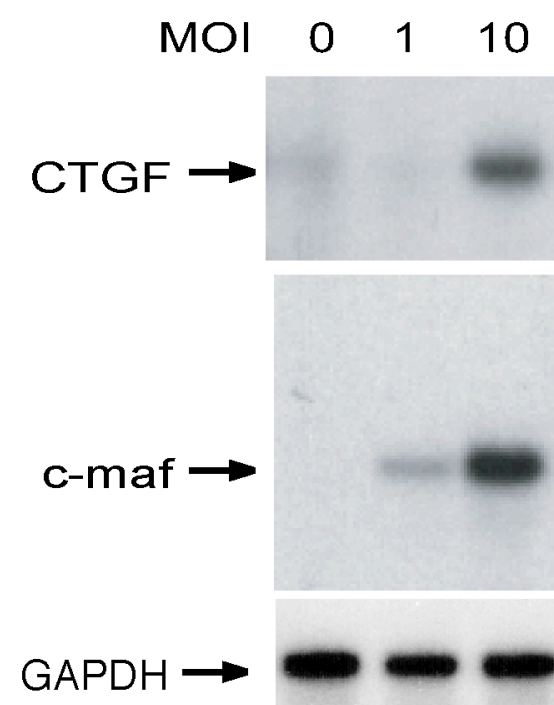

B

$\begin{array}{ccccc}\text { MOI } & 0 & 1 & 10 \quad 20 \\ \text { CTGF } & & & - \\ \text { C-Maf } & & & =\end{array}$

C c-Maf gene +/+ -/- $-/-$ Ad/c-maf - - + CTGF GAPDH

Fig. 2 
A

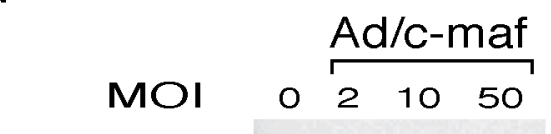

TGF- $\beta$

CTGF $\rightarrow$

c-maf

$\rightarrow$

GAPDH
B

TGF- $\beta(\mathrm{ng} / \mathrm{ml}) \quad 0 \quad 0.1 \quad 10 \quad$ Kidney

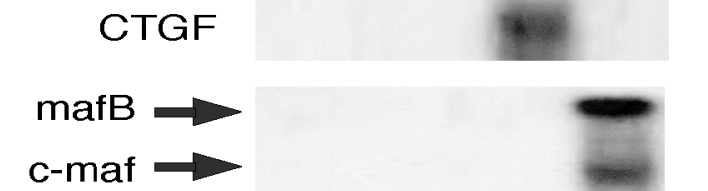

C

$\begin{array}{ccccc}\text { TGF- } \beta(\mathrm{ng} / \mathrm{ml}) & 0 & 0.1 & 10 & \mathrm{c}-\mathrm{Maf} / \mathrm{Mal} \\ \text { CTGF } & & & & \\ \text { C-Maf } & & & \end{array}$

Fig. 3 
A

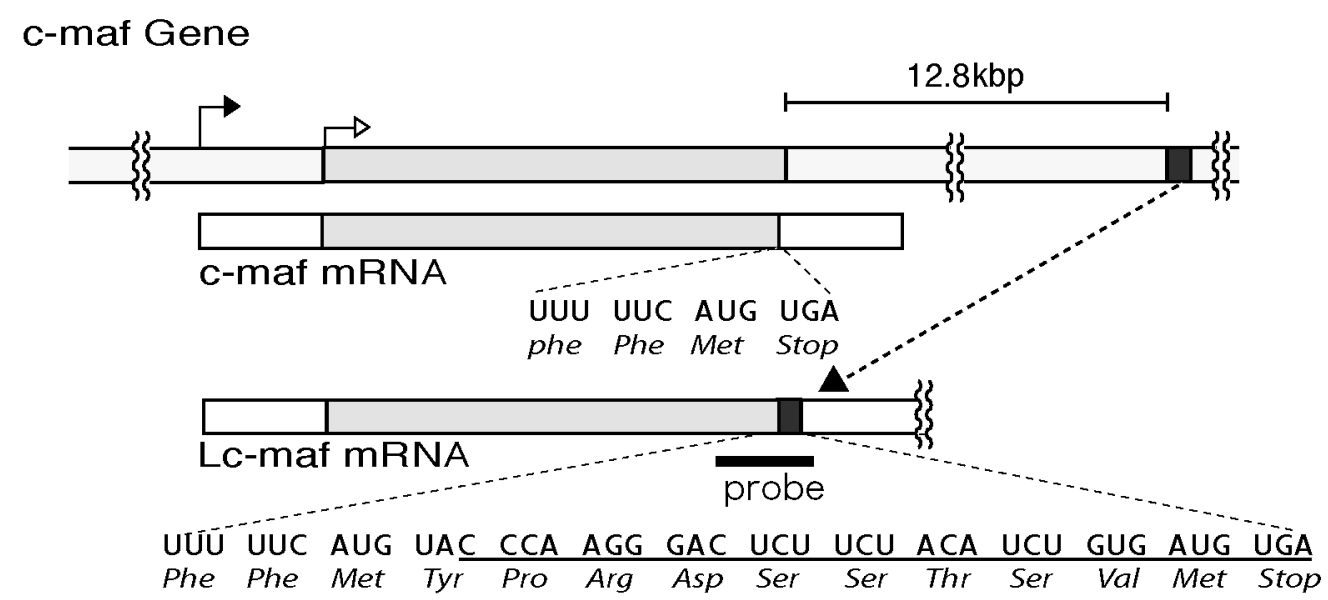

B

Liv $\mathrm{Br}$ Kid Mus BMP

Lc-maf

c-maf

Fig. 5 


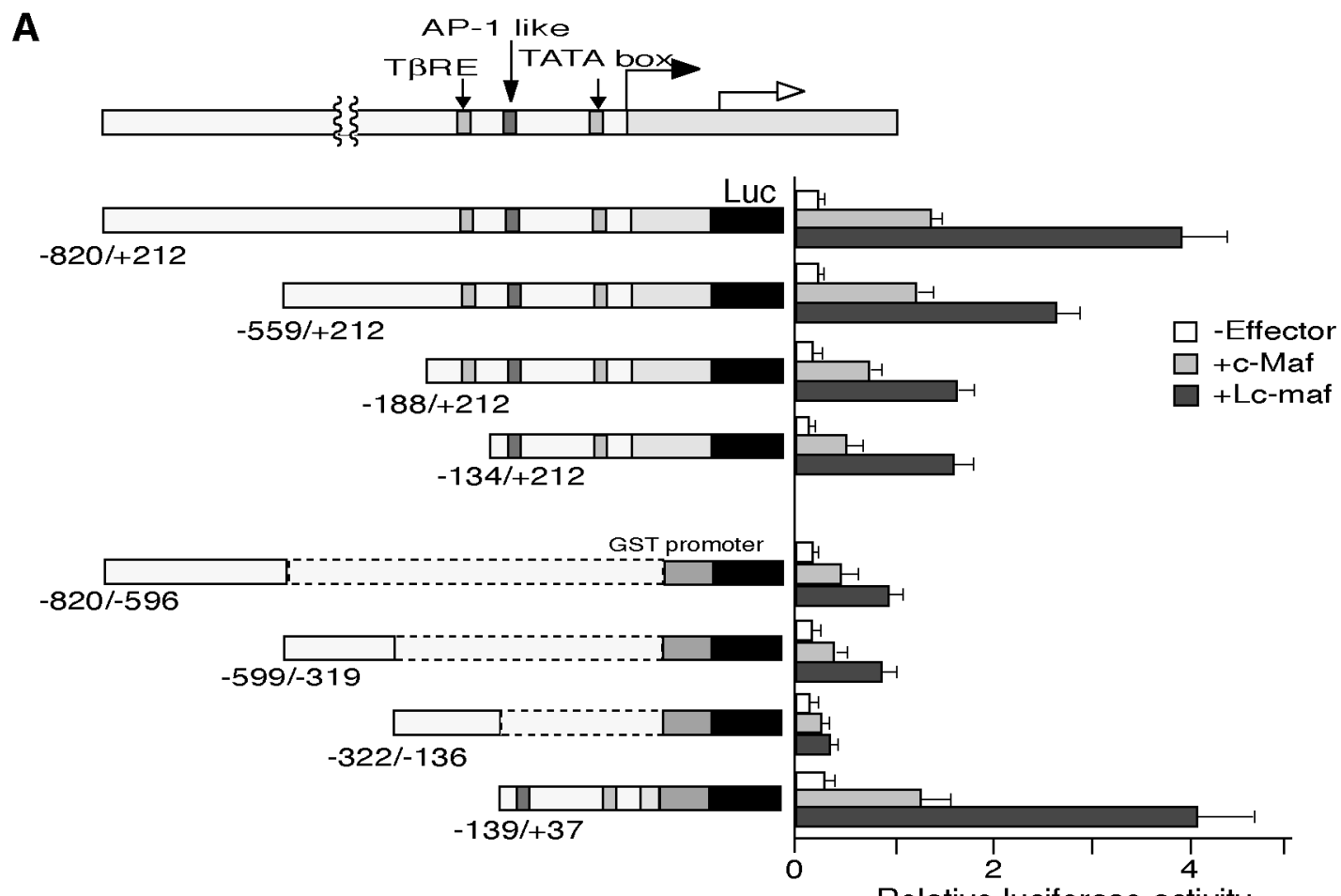

B

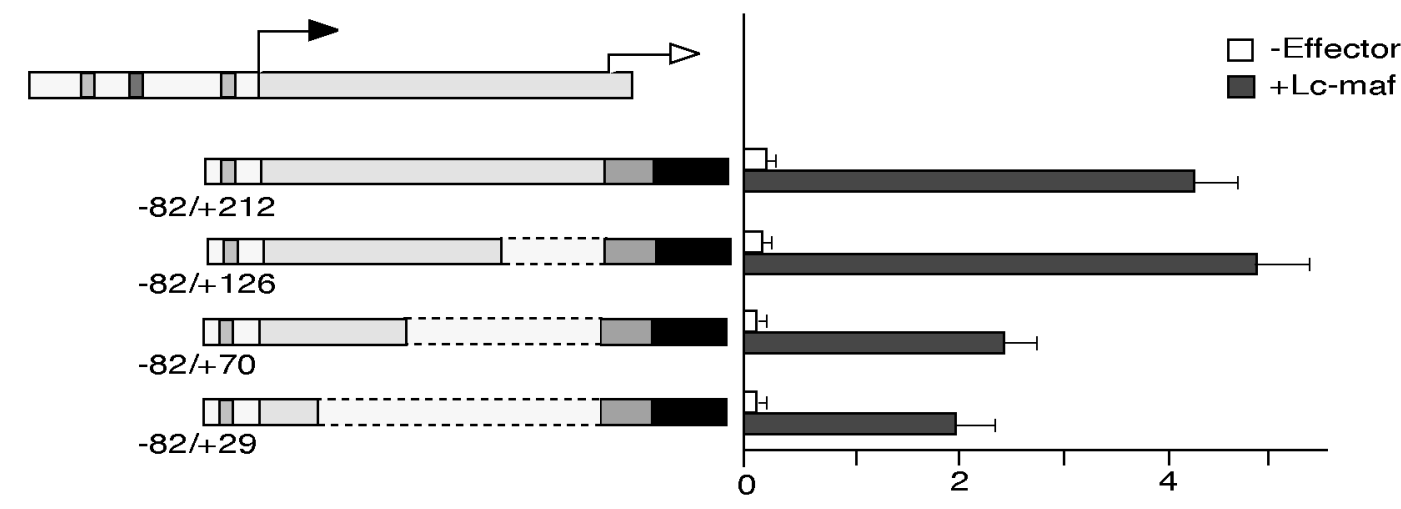

Relative luciferase activity

Fig. 5 


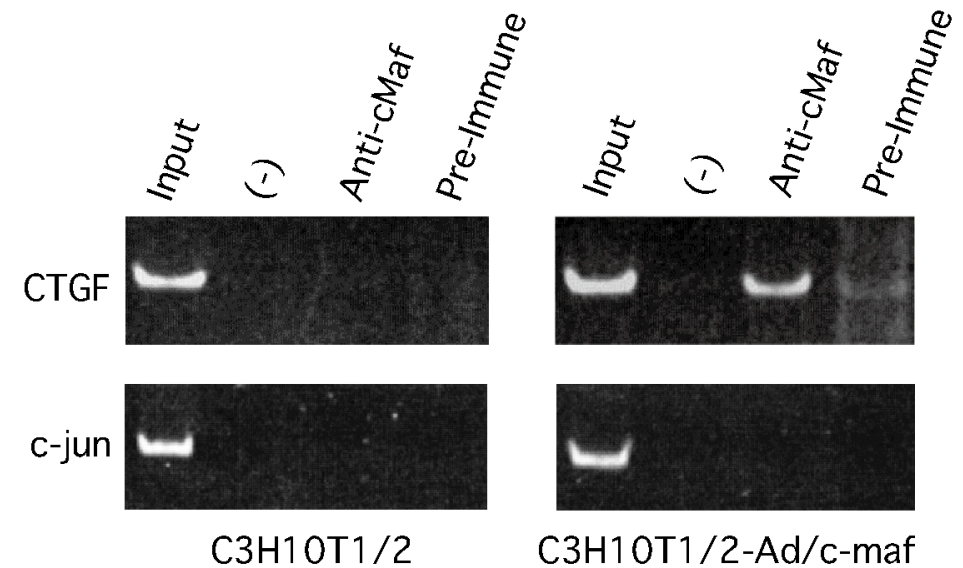

Fig. 6 